\title{
Epigenetic reprogramming during early development in mammals
}

\author{
Fátima Santos and Wendy Dean \\ Laboratory of Developmental Genetics and Imprinting, Developmental Genetics Programme, \\ The Babraham Institute, Cambridge CB2 4AT, UK \\ Correspondence should be addressed to Wendy Dean; Email: wendy.dean@bbsrc.ac.uk
}

\begin{abstract}
Epigenetic modifications serve as an extension of the information content by which the underlying genetic code may be interpreted. These modifications mark genomic regions and act as heritable and stable instructions for the specification of chromatin organisation and structure that dictate transcriptional states. In mammals, DNA methylation and the modification of histones account for the major epigenetic alterations. Two cycles of DNA methylation reprogramming have been characterised. During germ cell development, epigenetic reprogramming of DNA methylation resets parent-of-origin based genomic imprints and restores totipotency to gametes. On fertilisation, the second cycle is triggered resulting in an asymmetric difference between parental genomes. Further epigenetic asymmetry is evident in the establishment of the first two lineages at the blastocyst stage. This differentiative event sets the epigenetic characteristics of the lineages as derivatives of the inner cell mass (somatic) and trophectoderm (extra-embryonic). It is the erasure and subsequent re-tracing of the epigenetic checkpoints that pose the most serious obstacles to somatic nuclear transfer. Elaboration of the mechanisms of these interactions will be invaluable in our fundamental understanding of biological processes and in achieving substantial therapeutic advances.

Reproduction (2004) 127 643-651
\end{abstract}

\section{Introduction}

This year marks the twentieth anniversary of the early work from the laboratories of Surani and Solter (Surani et al. 1984, McGrath \& Solter 1984) that led to the biological principle of pronuclear non equivalency at fertilisation. These studies set the scene for a mechanistic explanation of the differential and complimentary potentials of gametes. In brief, monoparental mouse embryos generated by micromanipulation manifest strict opposing phenotypes. Gynogenetic embryos (diploid maternal) characteristically are growth restricted and fail to derive a functional placenta. In contrast, androgenetic embryos (diploid paternal) while profoundly growth retarded, hyperproliferate extra-embryonic tissues. The essence of this difference was the understanding that during maturation of gametes there is marking of specific regions of the genome for later differential expression (Surani et al. 1990). This parent-oforigin mark, or genomic imprint, has led to the realisation that epigenetic differences play an absolutely critical role in the re-establishment of totipotency of the zygote (McGrath \& Solter 1984). Furthermore, these early differences are reflected later during the establishment of the first lineages at the blastocyst stage. Thereafter, epigenetic information is faithfully and heritably reiterated according to their early lineage origin.

The focus of this review is to recount the current state of understanding of epigenetic modifications and some of the activities thought to be involved in the establishment of epigenetic marks during preimplantation development in mammals. The consequence of reprogramming of somatic nuclei through investigations of epigenetic marking in cloned embryos during this early phase of development is highlighted.

\section{DNA methylation}

Epigenetic reprogramming can be defined as any meiotic or mitotic alteration that does not result in a change in DNA sequence but will have a significant impact on the development of the organism (Russo et al. 1996). The most prominent form of epigenetic alteration in mammals is the symmetric methylation of cytosine in the $5^{\prime}$ position in $\mathrm{CpG}$ dinucleotides. DNA methylation can be thought to have a twofold biological significance, gene regulation and structural fidelity (Bestor 2000, Robertson \& Wolffe 2000). DNA methylation has been firmly established as 
playing a critical part in transcriptional repression. This has been described in the context of development and establishes the allele-specific expression status in many imprinted loci through differential DNA methylation of parental alleles (i.e. differential methylated regions, DMRs) (Li et al. 1993). DNA methylation has been implicated in 'genome defence' associated with the silencing of parasitic retrotransposons (Yoder et al., 1997) and to a function in the maintenance of the structural integrity of chromosomes and prevention of chromosomal rearrangements (Chen et al. 1998).

\section{DNA methylation reprogramming}

Mammalian development is characterised by bimodal DNA methylation reprogramming that occurs initially during germ cell development and then during preimplantation (Fig. 1) (Reik \& Walter 2001a). Primordial germ cells (PGCs) enter the developing germinal ridge and begin differentiation and expansion. At this time, the highly methylated PGCs undergo rapid genome-wide demethylation such that by day 12.5 most of the methylation is lost (Reik \& Walter 2001b). This reprogramming phase coincides with the erasure and resetting of parentof-origin specific marks that include DNA methylation of imprinted DMRs associated with allele-specific gene expression (Tucker et al. 1996). The exact timing of de novo methylation has not been firmly established but is initiated in males at $\sim 14.5 \mathrm{dpc}$ (days post-coitum) and thereafter in females such that the mature gametes of both sexes will eventually become highly methylated.

The second phase of methylation reprogramming occurs between fertilisation and formation of the blastocyst. On fertilisation a rapid paternal-specific asymmetric loss of methylation is observed (Mayer et al. 2000, Dean et al. 2003). This process takes place in the absence of transcription or DNA replication and is termed active demethylation. Thereafter, there is a step-wise decline in methylation until the morula stage (Dean et al. 2001, Santos et al. 2002). This decline occurs as a result of the absence of the primary DNA methyl transferase, Dnmt1, during DNA replication (Bestor 2000). Thus, the newly replicated strand fails to become methylated and the level of methyl cytosine per nucleus declines. This replicationdependent loss of DNA methylation is referred to as passive demethylation (Rougier et al. 1998). The initiation of the de novo methylation occurs after the fifth cell cycle and coincides with the time of the first differentiative event. The establishment of the first two cell lineages results in yet another significant asymmetry. The inner cell mass (ICM), which gives rise to all the tissues of the adult, becomes hypermethylated, while the trophectoderm (TE), that forms most of the structure of the placenta, is undermethylated (Dean et al. 2001, Santos et al. 2002). This differential is maintained and reflected in highly methylated somatic tissues and the distinctively hypomethylated extra-embryonic tissues of the placenta. Among the somatic tissues that derive from the ICM are the highly methylated PGCs which arise $\sim$ day 7 in the extraembryonic mesoderm of the developing embryo (Ginsburg et al. 1990). Their migration via the allantois to the developing germinal ridges, where they will eventually differentiate into mature gametes, completes the cycle of epigenetic reprogramming.

Despite the genome-wide decline in DNA methylation, certain sequences remain refractory to the general demethylation during preimplantation development. Of special interest is the observation that DMRs of imprinted genes and certain classes of repeat sequences are exempt from these demethylation events (Reik \& Walter 2001a). Methylation analysis of bisulphite-treated DNA isolated from oocytes and sperm suggest that intracisternal A particles maintain their methylation (Lane et al. 2003). This interesting observation begs the question of the role of both active and passive demethylation during preimplantation development. The significance of active demethylation has been at the centre of considerable debate in the field of epigenetics. Two hypotheses have been tabled to explain the need for active demethylation. Paternalspecific loss of methylation may simply be required to allow for generalised de-repression of paternal alleles to accommodate the minor transcriptional burst at the end of the first cell cycle (Aoki et al. 1997). Perhaps more provocative is the implication of active demethylation in the resolution of parent-offspring conflict that is exerted via imprinted gene expression (Moore \& Haig 1991). This proposal embodies an evolutionary principle purporting that each parent battles to maximise their own genetic fitness by ensuring the transmission of their genetic legacy. Thus, paternal interests seek to exert control over genes that maximise growth and survival of individual offspring while maternal interests must try to moderate the growth of single individuals for the benefit of the entire litter. This mediation might be possible through selective methylation and demethylation of growth regulatory genes. In this regard many imprinted genes identified to date have growth regulatory functions or influence postnatal suckling behaviour (Beechey et al. 2003).

A number of predictions arise from this hypothesis. In support of the notion that active demethylation arose in the context of imprinting in mammals is the observation that neither Xenopus (Stancheva et al. 2002) nor zebra fish (Macleod et al. 1999) show active demethylation. Furthermore, it would be expected that active demethylation should be conserved in mammals and should take place in the first cell cycle rather than immediately prior to zygotic genome activation. Active paternal-specific demethylation has been confirmed in several other mammals including the rat, pig and bovine, and, partially, in sheep (Dean et al. 2003, F Santos, unpublished data). However, what remains illusive is the identity of this active demethylase. In order to learn more about this process we have undertaken an in-depth study of the first cell cycle of the mouse with respect to paternal-specific 

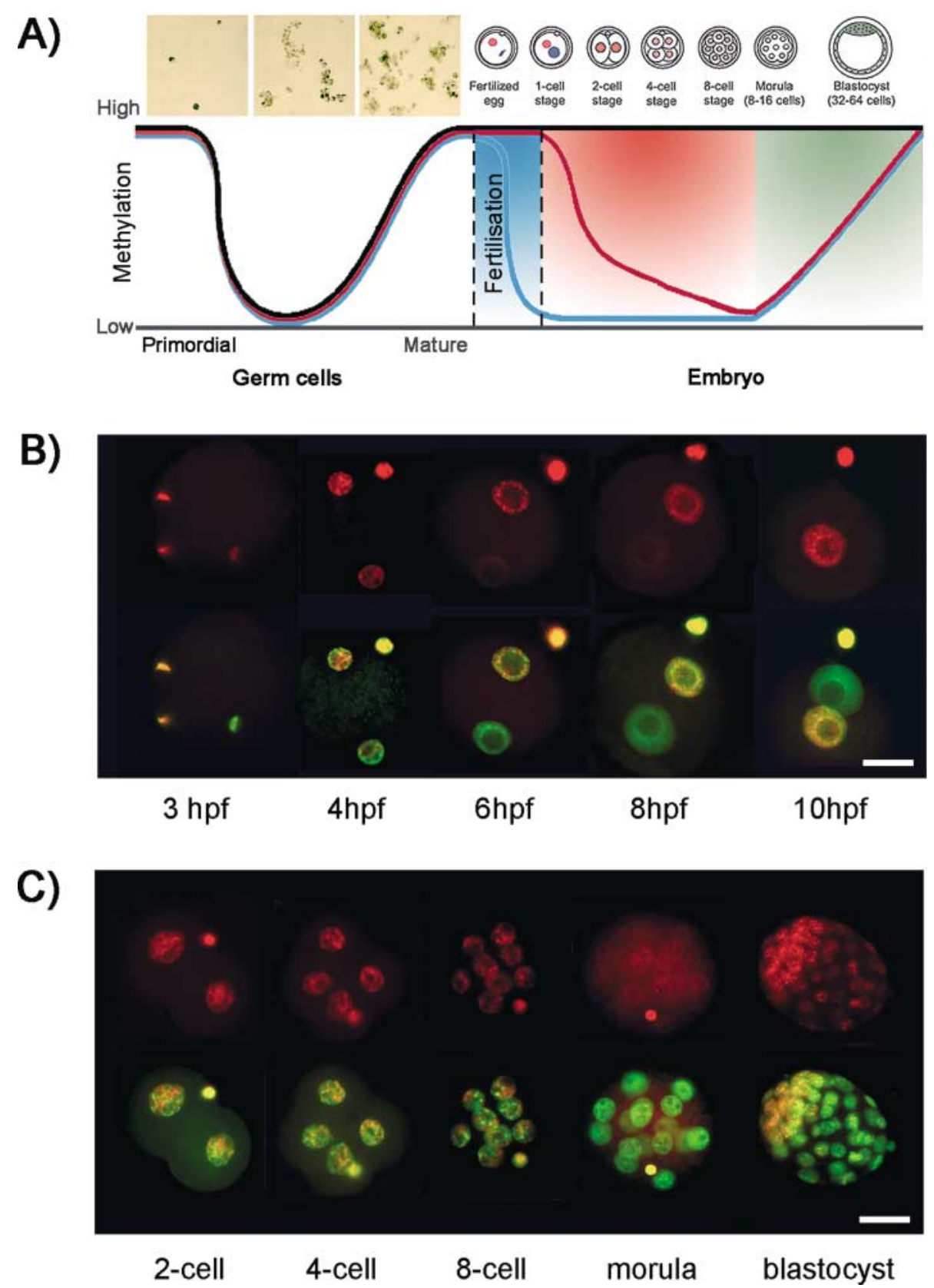

Figure 1 Methylation reprogramming during mouse development. The diagram depicts methylation levels of various classes of genes during germ cell and embryonic development (methylated imprinted genes (black line) and non-imprinted genetic sequences (red, maternal; blue, paternal)). (A) Highly methylated primordial germ cells enter the germinal ridge and undergo loss and reacquisition of methylation during their expansion phase. Examples of these cells (day 11.5, 13.5 and 14.5) stained for alkaline phosphatase, a PGC marker, are pictured above. The horizontal time axis and the vertical axis indicating the relative methylation levels are not to scale (modified from Reik \& Walter $2001 \mathrm{a}$ ). Genome-wide DNA methylation reprogramming is represented by indirect immunofluorescence of fertilised oocytes (B) and embryos (C) using an antibody to 5-methyl cytidine (5MeC). (B) Active demethylation: the first cell cycle. In the fertilised mouse oocyte a rapid and asymmetric loss of DNA methylation (red signal) can be observed in the male (green in lower merge panel) but not in the female pronucleus. Genome-wide loss of DNA methylation starts at sperm decondensation (left) and continues until it is undetectable in the paternal compartment (right), the process taking about $6 \mathrm{~h}$. Lower panels show a merge (yellow) between the DNA methylation (red) and DNA stain (green). hpf, hours post fertilisation. Scale bar $25 \mu \mathrm{m}$ (Santos et al. 2002). (C) Passive demethylation phase. From the 2-cell stage (left) to the morula (right) the DNA methylation (red) is passively lost due to the exclusion of DNA methyltransferase 1 (Dnmt1) from the nucleus. By the blastocyst stage lineagespecific de novo methylation is apparent with the inner cell mass (ICM) being highly methylated (red) while the trophectoderm remains hypomethylated. Mouse embryos are depicted. Lower panels show a merge (yellow) between the DNA methylation (red) and DNA stain (green). Scale bar $25 \mu \mathrm{m}$ (Santos et al. 2002). 
demethylation using an exquisitely sensitive and specific antibody to 5-methyl cytidine (5MeC) (Reynaud et al. 1992). To achieve this high-resolution profile, materials were generated by in vitro fertilisation (IVF). Thus, control over very early post-fertilisation events was possible and both an estimate of the rate and a more exact time point for demethylation could be determined. At fertilisation, the male and female gametes are at different stages of meiotic maturation (Fig. 2A). The female is arrested in MII metaphase awaiting the signal for the completion of meiosis. The male, while haploid, is complexed in a nearly inert toroidal configuration unique to the presence of protamines (Braun 2001). Thus, in order to restore diploid complement to the zygote extensive remodelling must occur. This involves decondensation and nucleoprotamine exchange for nucleohistone. Nucleohistone exchange has
A)

Oocyte
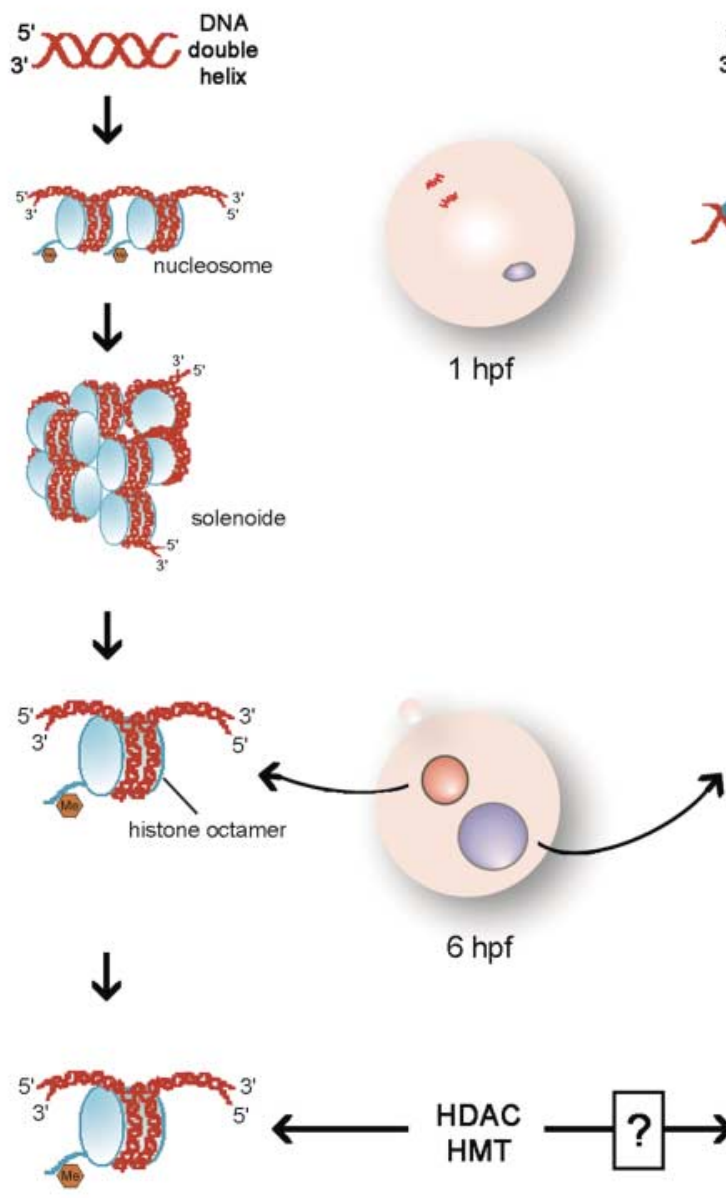

$6 \mathrm{hpf}$

HDAC

HMT

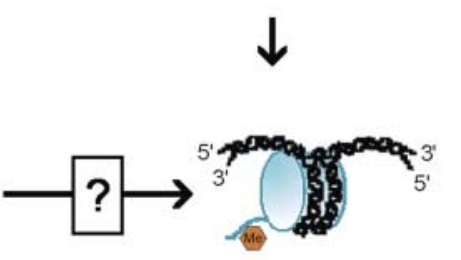

Sperm

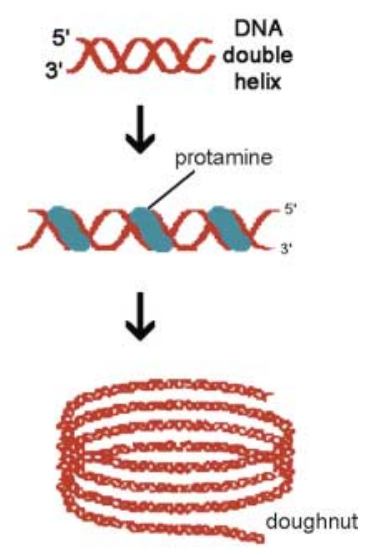

$\downarrow$

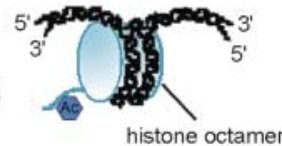

histone octamer

Methylated histone $\mathrm{NH}_{2}$-terminus

Acetylated histone $\mathrm{NH}_{2}$ terminus

B)
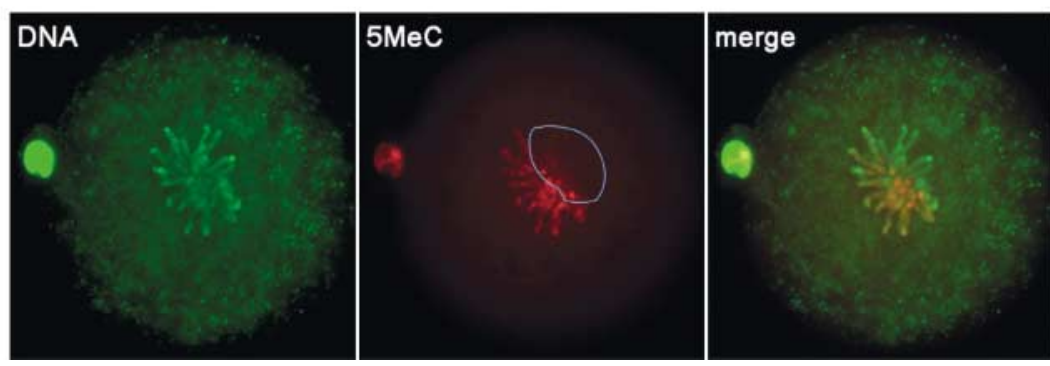
already taken place by the earliest time that antibody accessibility can be demonstrated in the decondensing mouse sperm (Perreault 1992, McLay \& Clarke 2003). Paternal-specific demethylation is observed as early as $3 \mathrm{~h}$ and completed within $6 \mathrm{~h}$ of IVF, several hours in advance of the initiation of DNA replication (Santos et al. 2002).

\section{Active demethylation: mechanisms and candidate activities}

In contrast to other vertebrate model organisms such as Xenopus, the small size and restriction on the number of female gametes poses considerable experimental challenges to some biochemical questions in the mouse. While isolation of this demethylase activity directly from mouse oocytes was not feasible, some experimental manipulation was accessible. To this end we attempted to titrate the demethylase activity in mouse oocytes using conditions permissive for polyspermy (Santos et al. 2002). Under appropriate conditions, up to five sperm can be matured in MII oocytes (Perreault 1992). Despite clear evidence of titration of other components required for pronuclear formation, demethylation of supernumerary male pronuclei took place without impediment (Fig. 3A). This unexpected result suggests that the oocytes were extremely well supplied with demethylase and that the specificity was tightly regulated as the female pronucleus remained unaffected.

Three basic mechanisms have been proposed for active demethylation. The first is the direct removal of the methyl group from the major groove. This 'snipping' away of the side group would require the breaking of carbon bonds and the production of methanol (Bhattacharya et al. 1999, Bird 2002). The second group of reactions would either specifically remove the methyl cytosine replacing it with a cytosine (Klimasauskas et al. 1994) or remove the CpG dinucleotide by nucleotide excision, a replication-dependent DNA repair process. A third possibility is hydrolytic deamination which results in the conversion of 5-methylcytosine to thymine that subsequently becomes repaired in the next replication cycle. Energetically this last option is highly unfavourable; however; this does open the field for the consideration of reactions that achieve deamination via enzymatic pathways.
Several candidates have been suggested for the active demethylase. Szyf and colleagues (Bhattacharya et al. 1999) reported an activity for methyl binding domain protein 2 (MBD2) which fulfilled the criterion for the active demethylase (Cedar \& Verdine 1999). Amidst much sceptical acceptance, MBD2 was thought to be a possible candidate in early embryos. Homozygous oocytes stained with antibody for $5 \mathrm{MeC}$ and were found to undergo active demethylation (Fig. 3B). Methyl binding domain protein 4 (MBD4), a uridine deglycosylase, has been proposed as a potential demethylase activity owing to its role in DNA repair (Hendrich \& Tweedie 2003). Fertilised oocytes homozygous for the deletion stained in a pattern indistinguishable from the control (Fig. 3B). This clear result suggested that MBD4 was not the activity responsible for active demethylation in the early mouse embryo (F Santos, unpublished data). Currently, other candidates are being pursued and we look forward with interest to the outcome of these studies.

Rehydration of sperm DNA during pronuclear maturation presents an intriguing proposition for paternalspecific active demethylation. The packaging of a haploid complement of DNA into the mature sperm is itself a feat of engineering triumph (Ward \& Coffey 1991). The extreme reduction in size due to the exclusion of water molecules provides an interesting source of 'free energy' on sperm decondensation. This process is energy producing and in the context of coupled reactions may be capable of supplying the energy needed for demethylation. ATP-coupled chromatin remodelling activities such as those provided by lymphoid-specific helicase (LSH), a member of the SNF/helicase family, are essential for normal murine development and are associated with establishing methylation patterns (Dennis et al. 2001). It is tempting to speculate that within this remodelling complex is an activity that functions at this early stage in a unique capacity as a DNA demethylase.

\section{Epigenetic reprogramming beyond the first cell cycle}

The decline in DNA methylation, largely from maternally derived sequences, continues in a step-wise fashion until the morula stage when very little signal can be detected

\footnotetext{
Figure 2 Asymmetric chromatin remodelling is a requisite for fertilisation. (A) At fertilisation the oocyte chromatin is organised in a somatic configuration with methylated (red) DNA complexed with histones (abundant methylation of $\mathrm{NH}_{2}$ termini). This status remains unchanged throughout female pronuclear maturation. In contrast, sperm chromatin is fully gametic, with protamines instead of histones, and highly dehydrated. Exchange of nucleoprotamine for nucleohistone has to occur during the first hour after fertilisation. Histones are acquired with acetylated lysines in the amino terminal tails. Meanwhile, DNA becomes demethylated (black) through an active process. The mature male pronucleus has only residual DNA methylation (centromeric region) and acetylated nucleosomes. This asymmetric distribution of chromatin organisation in the fertilised oocyte raises a number of interesting points. In order to achieve a somatic organisation of the male chromatin the transition will first require the removal of the acetylation group catalysed by histone deacetylases (HDACs), followed by the recruitment of specific histone methyltransferases (HMTs). Mechanistically, the inability to make this transition from acetylated to methylated histone may be the cause of the loss of DNA methylation specifically from the male pronucleus (chromatin in the zygote adapted from Braun, 2001). hpf, hours post fertilisation. (B) At syngamy, the maternal and paternal chromosomes align themselves in a unique configuration maintaining their parental associations. Maternal chromosomes show DNA methylation along the entire chromosome while paternal chromosomes (encircled) retain only centromeric methylation. This highlights the epigenetic differences between maternal and paternal chromosomes and points to interesting chromatin differences that may well remain throughout the preimplantation period of development.
} 
by $5 \mathrm{MeC}$ antibody staining (Rougier et al. 1998). This passive loss of methylation occurs as the oocyte form of the primary methyl transferase (Dnmt1o) is actively excluded from the nucleus despite the extraordinary abundance of the protein inherited in the cytoplasm (Howell et al. 2001). In a most remarkable and specific form of temporal regulation, the Dnmt1o is excluded except for a single cell cycle at the eight-cell stage. Interestingly, deletion of the Dnmt1o $5^{\prime}$ exon results in the abolition of imprinted methylation marks that cannot thereafter be restored (Dean \&
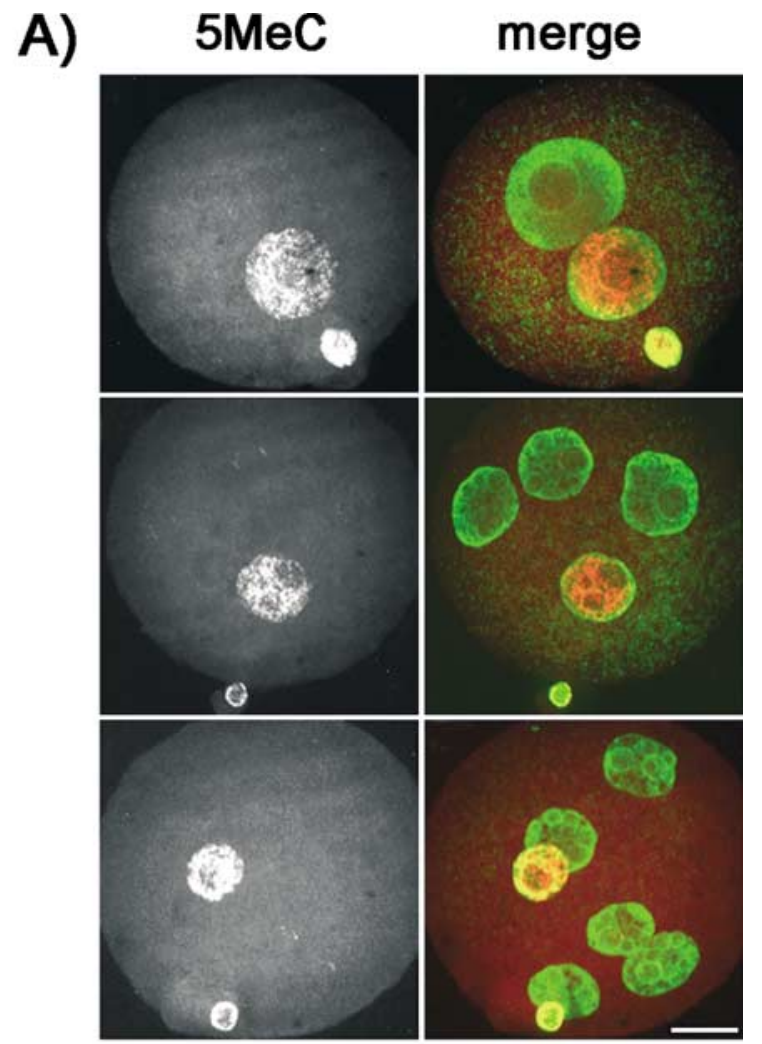

\section{B)}

$5 \mathrm{MeC}$

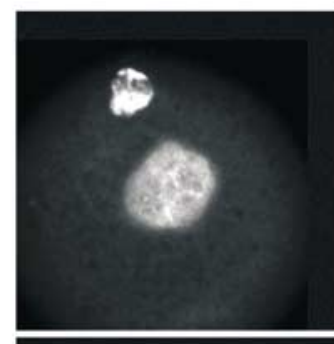

MBD2

MBD4

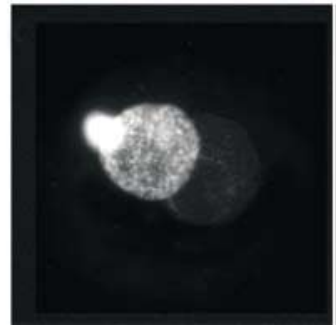

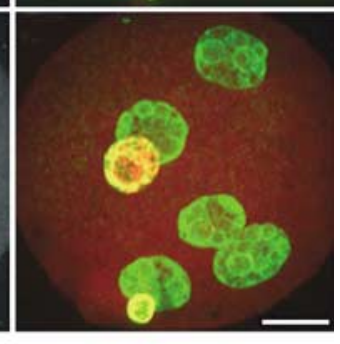

merge

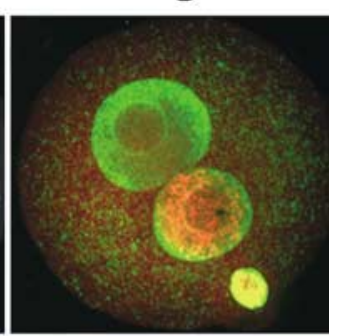

merge
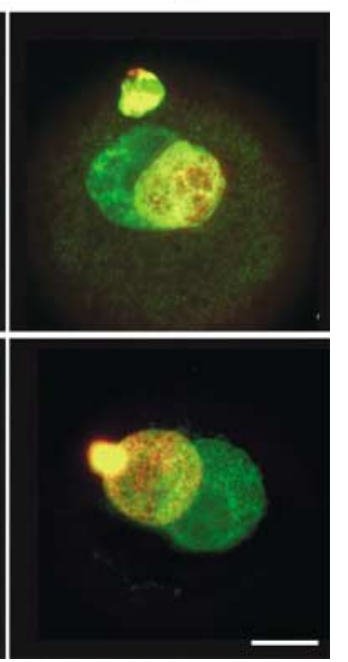

Ferguson-Smith 2001, Howell et al. 2001). What is special about the eight-cell stage? Perhaps the answer lies in the changes in totipotency that occur at this time when the blastomeres of the embryo exhibit different cellular features and are known to acquire an 'inside-outside identity', arguably the very first cell determination event (Johnson 1986). These changes lead to the formation of presumptive embryonic (inside; ICM) and presumptive extra-embryonic (outside; TE) lineages (Johnson 1986). By the 5th cell cycle the final phase of methylation reprogramming is initiated. De novo methylation is evident in both cell lineages of the blastocyst. However, it is the ICM that becomes hypermethylated in comparison with the TE. This establishes the asymmetric level of methylation seen in somatic cells as ICM derivatives and in the placenta as a TE derivative (Reik et al. 2003).

\section{Epigenetic remodelling in the early embryo: implication for somatic nuclear transfer}

Since the establishment of paternal-specific demethylation in the fertilised oocyte, the question of the associated chromatin structure and organisation permissive for this event has become the focus of our attention. In organisms which possess DNA methylation, an inter-reliance exists between these epigenetic marking systems to specify transcriptional states and structural features of chromatin (Strahl \& Allis 2000, Jenuwein \& Allis 2001).

The $5^{\prime}$ amino-terminal tails of the core histones of the nucleosome lie in the major groove of the double helix and can be covalently modified by post-translational addition including methylation, acetylation, phosphorylation, ubiquitination and ADP-ribosylation to lysine, serine and arginine residues. This led to the postulate that the interpretation of the underlying genetic code was, in part, achieved through the combinatorial modifications of key amino acid residues of core histones or a 'histone code' (Strahl \& Allis 2000, Turner 2000, Jenuwein \& Allis 2001). Switching between these modifications was associated with specific chromatin states and the transitions from

Figure 3 Active demethylation: mechanisms and candidate activities. (A) Polyspermic fertilisation of zona-free mouse oocytes. To evaluate whether the demethylation of the paternal allele was titratable, oocytes were exposed to capacitated sperm after removal of the zonae and incubated for $9.5 \mathrm{~h}$ prior to fixation and staining with antibody to $5 \mathrm{MeC}$. This procedure produced fertilised oocytes containing multiple extra male pronuclei. The results show some of these polyspermic fertilised embryos with one male pronucleus, and three and five male pronuclei. Titration of components necessary for pronuclear formation is evident as the size of the pronucleus is inversely proportional to the number of supernumerary male pronuclei (Santos et al. 2002). (B) Distribution of CpG methylation during pronuclear stages in the MBD2 and MBD4 null mouse fertilised oocytes using indirect immunofluorescence with $5 \mathrm{MeC}$ antibody. Demethylation of the male pronucleus was indistinguishable from that of appropriate controls. Scale bar $20 \mu \mathrm{m}$. 
active to inactive configurations. In general, methylation of residues is associated with transcriptional repression while acetylation is known to accompany actively transcribing regions. With this possibility in mind investigations have focussed intensively around residues of core histones that may adopt either modification. Thus a vast and expanding literature is evolving around the significance of chromatin modification of lysine 9 of histone $\mathrm{H} 3$ (H3-K9) (reviewed in Lachner et al. 2003).
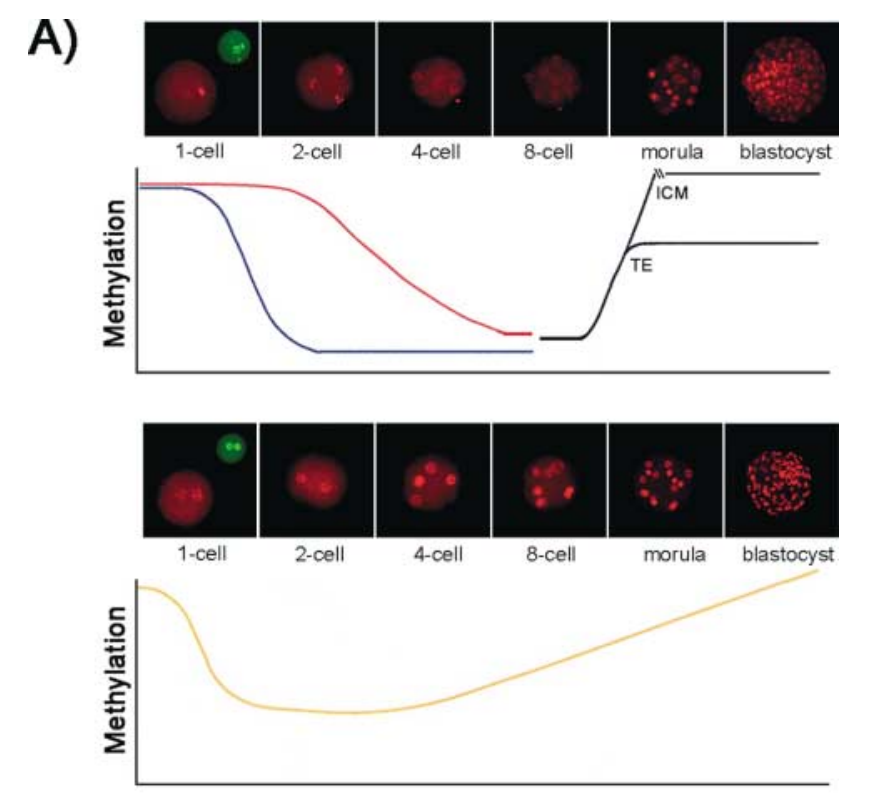

B)
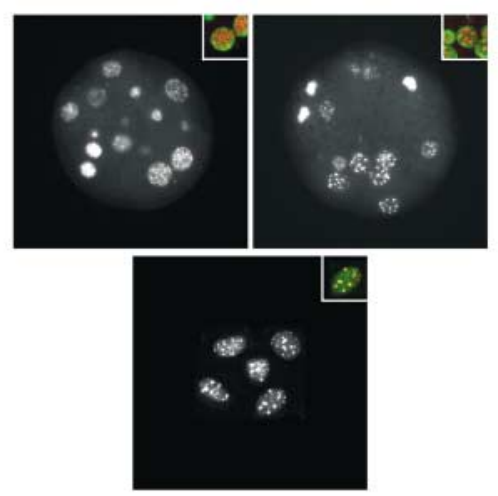

C)

Increased levels and abnormal organisation of DNA methylation and $\mathrm{H} 3-\mathrm{K} 9$ methylation patterns

Loss of epigenetic asymmetry between ICM and TE may explain placental abnormalities consistently observed on cloning
Given the asymmetric loss of DNA methylation from the paternal compartment, investigation of chromatin modifications in the one-cell fertilised oocytes is of particular interest (Arney et al. 2002, Cowell et al. 2002). Using a heterochromatin-specific antibody to dimethylated $\mathrm{H} 3-\mathrm{K} 9$ (anti- $\alpha-4 \mathrm{x}-\mathrm{methH} 3-\mathrm{K} 9$ ), it was demonstrated that the decondensing sperm and maturing male pronuclei are negative for this modification (Reik et al. 2003). In contrast, the female pronucleus stains intensely for the same configuration, which remains throughout the first cell cycle. Despite this histone methylation asymmetry, acetylated H3-K9 was found to be abundant in both pronuclei, in agreement with earlier reports (Adenot et al. 1997).

Remodelling of the sperm nucleus is a requisite for fertilisation (Fig. 2A). Exchange of nucleoprotamine for nucleohistone occurs during the first hour after fertilisation (Perreault 1992). Acetylation of histones takes place in the cytoplasm mediated by histone acetyl transferases (HATs) and thus histones are acquired with acetylated lysines in the amino terminal tails (Turner 2000). Methylation takes place in the nucleus mediated by histone methyl transferases (HMTs). The transition first involves the removal of the acetylation group catalysed by histone deacetylases (HDACs). Thus the smooth transition from an active (acetylated) to an inactive and potentially silent (methylated) chromatin configuration requires a series of specific enzymatic activities. At present, no lysine-specific histone demethylase activities have been identified and thus the only mechanism of reactivation of silent loci may be by replacement of histones (Lachner et al. 2003).

The asymmetric distribution of chromatin organised in the fertilised oocyte raises a number of interesting points. The heterochromatin-specific antibody is known to associate with peri- and centromeric satellite sequences and other substantial stretches of heterochromatin throughout

Figure 4 Genome-wide DNA methylation in IVF produced and cloned bovine embryos. (A) In bovine the paternal genome (blue) undergoes active demethylation while the maternal genome (red) is passively demethylated until the 8-cell stage when de novo methylation (black line) is observed (upper panel). At the blastocyst stage there is an asymmetric distribution of DNA methylation between the ICM (embryonic) and TE (extra-embryonic) lineages. This pattern is altered on somatic nuclear transfer. Cloned embryos (lower panel) show active but not passive demethylation activity. However, precocious de novo methylation is observed by the 4-cell stage suggesting that it may occur as early as the second cell cycle. (B) Organisation of DNA methylation in normal and cloned bovine embryos. Between the 8 - and 16-cell stage a heterogeneous population of nuclei is observed in control embryos (left panel). Cloned embryos (right panel) are highly homogeneous with a methylation pattern strongly reminiscent of the donor nuclei (lower panel) (Dean et al. 2001). (C) Cloned embryos fail to achieve the asymmetry of both DNA and $\mathrm{H3}$ K9 methylation characteristic of un-manipulated control blastocysts. Aberrant hypermethylation of the trophectoderm in cloned embryos may be an early marker of the placental abnormalities frequently reported. The figure depicts the merged channels of DNA and H3-K9 methylation (modified from Santos et al. 2003). 
the genome (Peters et al. 2002). Ordinarily, the presence of methH3-K9 is associated with transcriptional repression. The conflict arises from the observation that although both the male and female pronuclei are transcriptionally silent, only the female has chromatin in a form associated with gene repression. A minor burst of transcriptional reactivation occurs at the end of the first cell cycle with paternally derived alleles expressed in advance (Aoki et al. 1997). These discrepancies suggest that the codified interpretation of histone modifications may be specific and distinctive in gametes and embryos, as compared with somatic cells where totipotency has been progressively restricted.

It is intriguing that the female pronucleus remains DNA methylated and possesses methyl histone modifications suggestive of a mechanistic link between these two epigenetic systems. This suggests at least two possible interpretations. Either the chromatin organisation of the female pronucleus is resistant to an activity found in both compartments or the male is specifically susceptible and targeted for demethylation.

\section{Epigenetic reprogramming on somatic nuclear transfer}

The strict progression of events that ordinarily take place and the precise and asymmetric outcomes of reprogramming highlight some epigenetic resetting features that pose a potential obstacle to somatic nuclear transfer (Wade \& Kikyo 2002). Must the highly methylated (DNA) nucleus of a differentiated somatic cell undergo reprogramming on cloning in order to develop to term? If so, how is the cellular memory system that ensures fidelity of the differentiated cell type circumvented? Does the oocyte, primed to remodel nucleoprotamine, recognise the chromatin configuration of the somatic cell and establish an epigenetic state equivalent to that of the fertilised oocyte?

We have begun to investigate some of these questions associated with somatic nuclear transfer in mammals by focusing attention on cloned preimplantation stage embryos. Owing to the persistent difficulties encountered when attempting cloning in the mouse, we have exploited the fact that somatic nuclear transfer in cattle is well established. Characterisation of 5-methyl cytosine biology in bovine preimplantation embryos revealed active demethylation of the paternal pronucleus, passive demethylation during the 2- and 4-cell stage and de novo methylation at the 10- to 16-cell stage (Fig. 4A). At the blastocyst stage, the ICM is hypermethylated compared with the trophectoderm, in a pattern reminiscent of the mouse (Dean et al. 2001). Investigation into the pericentromeric heterochromatin using antibody to $\mathrm{H} 3-\mathrm{K} 9$ methylation revealed co-ordinate modulation together with the DNA methylation suggesting a mechanistic relationship between these two epigenetic systems (Dean et al. 2001, Santos et al. 2003).
Epigenetic profiles of cloned embryos produced from two different donor sources indicated that quantitative and qualitative deregulation of DNA and histone methylation occurred in reconstructed embryos. Perhaps the most striking aspect of these embryos was the resemblance of the pattern of DNA methylation and heterochromatic organisation to that of the donor cells (Fig. 4B) and the loss of lineage based asymmetric patterns of epigenetic marks (Fig. 4C). Furthermore, there was a correlation between normal epigenotype and rate of development to the blastocyst stage indicating it was predictive for normality (Santos et al. 2003).

These results reinforce the fact that one of the key features of somatic nuclear transfer will be the establishment of conditions that allow for erasure of heritable memory systems which are a hallmark feature of differentiated cell types (Wade \& Kikyo 2002). In addition, selection of donor populations that are intrinsically more reprogrammable by the oocyte cytoplasm will be essential (Solter 2000). These criteria are at present described only by epigenetic profiling using antibodies; however, they are powerful tools that permit rapid screening with a minimum of tissue, a persistent challenge to the mammalian developmental epigeneticist.

Further investigations using genetically defined mutations will be essential to tease apart the complex features of the dynamically evolving organisation of chromatin in germ cells, embryos and their differentiated antecedents. We anticipate a deluge of information in the next few years that will begin to explain the interrelated functions of epigenetic marks and the instructions they provide for the seamless unfolding of cellular function and developmental processes.

\section{Acknowledgements}

The work carried out by the authors takes place in the Laboratory of Developmental Genetics and Imprinting, Babraham Institute. We would like to thank Wolf Reik for allowing us to embark on this exciting research area. We apologise in advance that many original references have been omitted owing to space restrictions. This work is funded by the BBSRC and MRC.

\section{References}

Adenot PG, Mercier Y, Renard JP \& Thompson EM 1997 Differential $\mathrm{H} 4$ acetylation of paternal and maternal chromatin precedes DNA replication and differential transcriptional activity in pronuclei of 1-cell mouse embryos. Development 124 4615-4625.

Aoki F, Worrad DM \& Schultz RM 1997 Regulation of transcriptional activity during the first and second cell cycles in the preimplantation mouse embryo. Developmental Biology 181 296-307.

Arney KL, Bao S, Bannister AJ, Kouzarides T \& Surani MA 2002 Histone methylation defines epigenetic asymmetry in the mouse zygote. International Journal of Developmental Biology $\mathbf{4 6}$ 317-320.

Beechey CV, Cattanach BM, Blake A \& Peters J 2003 MRC Mammalian Genetics Unit, Harwell, Oxfordshire. World Wide Web Site Mouse Imprinting Data and References (http://www.mgu.har.mrc. ac.uk/imprinting/imprinting.html). 
Bestor TH 2000 The DNA methyltransferases of mammals. Human Molecular Genetics 9 2395-2402.

Bhattacharya SK, Ramchandani S, Cervoni N \& Szyf M 1999 A mammalian protein with specific demethylase activity for mCpG DNA. Nature 397 579-583.

Bird A 2002 DNA methylation patterns and epigenetic memory. Genes and Development 16 6-21.

Braun RE 2001 Packaging paternal chromosomes with protamine. Nature Genetics 28 10-12.

Cedar H \& Verdine GL 1999 Gene expression. The amazing demethylase. Nature 397 568-569.

Chen RZ, Pettersson U, Beard C, Jackson-Grusby L \& Jaenisch R 1998 DNA hypomethylation leads to elevated mutation rates. Nature 395 89-93.

Cowell IG, Aucott R, Mahadevaiah SK, Burgoyne PS, Huskisson N, Bongiorni S et al. 2002 Heterochromatin, HP1 and methylation at lysine 9 of histone $\mathrm{H} 3$ in animals. Chromosoma $11122-36$.

Dean W \& Ferguson-Smith A 2001 Genomic imprinting: mother maintains methylation marks. Current Biology 11 R527-R530.

Dean W, Santos F, Stojkovic M, Zakhartchenko V, Walter J, Wolf E \& Reik W 2001 Conservation of methylation reprogramming in mammalian development: aberrant reprogramming in cloned embryos. PNAS 98 13734-13738.

Dean W, Santos F \& Reik W 2003 Epigenetic reprogramming in early mammalian development and following somatic nuclear transfer. Seminars in Cell and Developmental Biology 14 93-100.

Dennis K, Fan T, Geiman T, Yan Q \& Muegge K 2001 LSH a member of the SNF2 family, is required for genome-wide methylation. Genes and Development 15 2940-2944.

Ginsburg M, Snow MH \& McLaren A 1990 Primordial germ cells in the mouse embryo during gastrulation. Development $\mathbf{1 1 0}$ 521-528.

Hendrich B \& Tweedie S 2003 The methyl-CpG binding domain and the evolving role of DNA methylation in animals. Trends in Genetics 19 269-277.

Howell CY, Bestor TH, Ding F, Latham KE, Mertineit C, Trasler JM \& Chaillet JR 2001 Genomic imprinting disrupted by a maternal effect mutation in the Dnmt1 gene. Cell 104 829-838.

Jenuwein T \& Allis CD 2001 Translating the histone code. Science 293 1074-1080.

Johnson MH 1986 Manipulation of early mammalian development: what does it tell us about cell lineages? Developmental Biology 4 279-296.

Klimasauskas S, Kumar S, Roberts RJ \& Cheng X 1994 Hhal methyltransferase flips its target base out of the DNA helix. Cell $\mathbf{7 6}$ 357-369.

Lachner M, O'Sullivan RJ \& Jenuwein T 2003 An epigenetic road map for histone lysine methylation. Journal of Cell Science $\mathbf{1 1 6}$ $2117-2124$

Lane N, Dean W, Erhardt S, Hajkova P, Surani A, Walter J \& Reik W 2003 Resistance of IAPs to methylation reprogramming may provide a mechanism for epigenetic inheritance in the mouse. Genesis 35 88-93.

Li E, Beard C \& Jaenisch R 1993 Role for DNA methylation in genomic imprinting. Nature 366 362-365.

McGrath J \& Solter D 1984 Completion of mouse embryogenesis requires both the maternal and paternal genomes. Cell 37 179-183.

McLay DW \& Clarke HJ 2003 Remodelling the paternal chromatin at fertilization in mammals. Reproduction 125 625-633.

Macleod D, Clark VH \& Bird A 1999 Absence of genome-wide changes in DNA methylation during development of the zebra fish. Nature Genetics 23 139-140.

Mayer W, Niveleau A, Walter J, Fundele R \& Haaf T 2000 Demethylation of the zygotic paternal genome. Nature 403 501-502.
Moore T \& Haig D 1991 Genomic imprinting in mammalian development: a parental tug-of-war. Trends in Genetics 7 45-49.

Perreault SD 1992 Chromatin remodeling in mammalian zygotes. Mutation Research 296 43-55.

Peters AH, Mermoud JE, O'Carroll D, Pagani M, Schweizer D, Brockdorff N \& Jenuwein T 2002 Histone H3 lysine 9 methylation is an epigenetic imprint of facultative heterochromatin. Nature Genetics 30 77-80.

Reik W \& Walter J 2001a Evolution of imprinting mechanisms: the battle of the sexes begins in the zygote. Nature Genetics 27 255-256.

Reik W \& Walter J 2001 b Genomic imprinting: parental influence on the genome. Nature Reviews. Genetics 2 21-32.

Reik W, Santos F, Mitsuya K, Morgan H \& Dean W 2003 Epigenetic asymmetry in the mammalian zygote and early embryo: relationship to lineage commitment? Philosophical Transactions of the Royal Society of London. Series B: Biological Sciences 358 1403-1409 discussion 1409.

Reynaud C, Bruno C, Boullanger P, Grange J Barbesti S \& Niveleau A 1992 Monitoring of urinary excretion of modified nucleosides in cancer patients using a set of six monoclonal antibodies. Cancer Letters $61255-262$.

Robertson KD \& Wolffe AP 2000 DNA methylation in health and disease. Nature Reviews. Genetics 11-19.

Rougier N, Bourc'his D, Gomes DM, Niveleau A, Plachot M, Paldi A \& Viegas-Pequignot E 1998 Chromosome methylation patterns during mammalian preimplantation development. Genes and Development 12 2108-2113.

Russo VEA, Martienssen RA \& Riggs AD 1996 Epigenetic Mechanisms of Gene Regulation. Cold Spring Harbor, NY: Cold Spring Harbor Laboratory Press.

Santos F, Hendrich B, Reik W \& Dean W 2002 Dynamic reprogramming of DNA methylation in the early mouse embryo. Developmental Biology 41 172-182.

Santos F, Zakhartchenko V, Stojkovic M, Peters A, Jenuwein T, Wolf E, Reik W \& Dean W 2003 Epigenetic marking correlates with developmental potential in cloned bovine preimplantation embryos. Current Biology 13 1116-1121.

Solter D 2000 Mammalian cloning: advances and limitations. Nature Reviews. Genetics 1 199-207.

Stancheva I, El-Maarri O, Walter J, Niveleau A \& Meehan RR 2002 DNA methylation at promoter regions regulates the timing of gene activation in Xenopus laevis embryos. Developmental Biology 243 155-165.

Strahl BD \& Allis CD 2000 The language of covalent histone modifications. Nature 403 41-45.

Surani MA, Barton SC \& Norris ML 1984 Development of reconstituted mouse eggs suggests imprinting of the genome during gametogenesis. Nature $\mathbf{3 0 8} 548-550$.

Surani MA, Kothary R, Allen ND, Singh PB, Fundele R, FergusonSmith AC \& Barton SC 1990 Genome imprinting and development in the mouse. Development. Supplement 89-98.

Tucker KL, Beard C, Dausmann J, Jackson-Grusby L, Laird PW, Lei H, Li E \& Jaenisch R 1996 Germ-line passage is required for establishment of methylation and expression patterns of imprinted but not of nonimprinted genes. Genes and Development 10 $1008-1020$.

Turner BM 2000 Histone acetylation and an epigenetic code. Bioessays 22 836-845.

Wade PA \& Kikyo N 2002 Chromatin remodeling in nuclear cloning. European Journal of Biochemistry 269 2284-2287.

Ward WS \& Coffey DS 1991 DNA packaging and organization in mammalian spermatozoa: comparison with somatic cells. Biology of Reproduction 44 569-574.

Yoder JA, Walsh CP \& Bestor TH 1997 Cytosine methylation and the ecology of intragenomic parasites. Trends in Genetics 13 335-340. 